\title{
AN ACCURATE MAPPING AND MONITORING GEOGRAPHICAL WATER STATUS USING WSN
}

\author{
Ashwini V. Mulay ${ }^{1}$ and Kailash J. Karande ${ }^{2}$
}

\begin{abstract}
The use of wireless sensors gives us a more precise way to get the water quality status at different places and $\mathrm{m}$ allow the enduring and easy deployment of monitoring stations at places of difficult access without the manual data retriev The proposed system can sense the different water parameters from water bodies continuously and send the real-time data the monitoring station using wireless technology to check the water quality. Using different sensors, this system will coll various parameters from water, such as $\mathrm{pH}$, turbidity and Dissolved Oxygen etc. The wireless sensor node consists processor, wireless communication module, external memory, power source and one or more sensors. The rapid developm of wireless sensor network (WSN) technology provides us a real-time data acquisition, transmission and data processing.

Keywords- WSN, pH, Turbidity, ARM processor
\end{abstract}

\section{INTRODUCTION}

Every living thing on earth needs water to survive. Water found in nature contains a number of impurities in varying amounts. As water is a universal solvent, water absorbs number of impurities in various ways like water runoff, etc. The impurities which are picked up by the water as suspended water sometimes make it more useful and suitable for drinking, but sometimes, it may make water totally harmful and unfit for drinking purpose and sometimes unfit for some other purposes. In some places such as in [1] describes that the pollutant effluent derived from mining sites has affected the underground water source such as wells, one of the important water source for people in rural area. Water quality is always an enormous issue, partly because of the tremendous growth of population \& urban expansion and development. The quality of water will not suffer, if people are diligent. So in a water supply project, quality of water is an important factor. Water quality analysis is the analysis of raw water to know various impurities present in it and for deciding the treatment of water.

Water quality affects because of surrounding conditions, environmental changes so there is necessity of regular testing. So continuous monitoring (in contrast to casual monitoring which is Manual collection and testing) of water quality will make sure us of its use. Currently, low resolution water quality monitoring is conducted, and water samples are manually collected at regular periods for chemical analysis and send the water samples to the laboratory. Such approaches are no longer considered efficient [9]. Continuous monitoring of surface water is imperative for continuous assessment of its ecological health, as well as anticipation.

Recently, wireless radio frequency technology has been widely used the solution in various surrounding application either to give wireless data transmission or remote controlling system. Hence, the Wireless Sensor Network (WSN) is an important and ideal technology platform for environmental remote monitoring infrastructure by looking at the benefits of its low power consumption and cost, smaller scale of size, networking, sensing flexibility, and mobility of nodes.

The system described in this paper can sense the water parameters using sensor node from the water bodies continuously and send the data to monitoring station called as a base station, using wireless technology. The system will use different sensors to collect various parameters from water bodies, such as $\mathrm{pH}$, dissolved oxygen, and turbidity, etc. The wireless sensor network (WSN) technology provides us a real-time data acquisition, transmission and processing as in [3][5]. There are different technologies which are used Wireless communication. A wireless sensor node has not only a sensing component, but also on-board processing, communication, and storage capabilities. Sensor node communicates with a base station BS using their wireless communication channel,

\footnotetext{
${ }^{1}$ Department Of Electronics and Telecommunication Engineering, SKN Sinhgad college of Engineering, Korti, Pandharpur. Tal- Pandharpur. Dist- Solapur. State-Maharashtra. India. Pin code- 413304.

${ }^{2}$ SKN Sinhgad college of Engineering Korti Pandharpur (INDIA)
} 
allowing them to disseminate their sensor data for remote processing, visualization, analysis, and storage systems. The base station is usually a PC with Graphic User Interface (GUI) for users to analyze water quality data or alarm automatically when water quality detected is below specific standards.

The rest of the paper is organized as follows. Working Principle is explained in section II. Hardware used for this system is described in section III. Explanation of Zigbee module is given in section IV. Algorithms are explained in section V. Practical implementation is presented in section VI. Concluding remarks are given in section VI.

\section{WORKING PRINCIPLE}

As water is needed to be tested regularly, the system should be monitor water parameters continuously. To monitor water quality in different sites in real time, an architecture constructed by distributed sensor nodes and base station is suggested in [2] [8]. Figure 1 shows an overview of system architecture. The system shows sensor node unit located at remote station and sensors are connected to sensor node unit to sense the physical parameter. At base station, PC is connected to the base station node unit which receives the data from the sensor node unit.

In our system there is focus on continuous monitoring of different water parameters in remote places or sources, collected water in school/college premises etc. For continuous monitoring of these physical parameters of water we can use wireless sensor network system. In Wireless Sensor Network system there is base station unit and sensor node unit. The Proposed water quality monitoring system is made up by base station and sensor nodes. The wireless sensor nodes are the vital element in a wireless sensor network (WSN). It keeps and executes the communication protocols and the data-processing algorithms. The sensor nodes are located in different places where we need to monitor water quality. The base station unit contains a wireless receiver and PC, where users can receive data from sensor nodes and analyze it. The base station and nodes are connected via WSN technology. Figure 1 shows the overall system. Figure 2 shows block diagram of sensor node unit. Figure 3 shows block diagram of base station node unit.

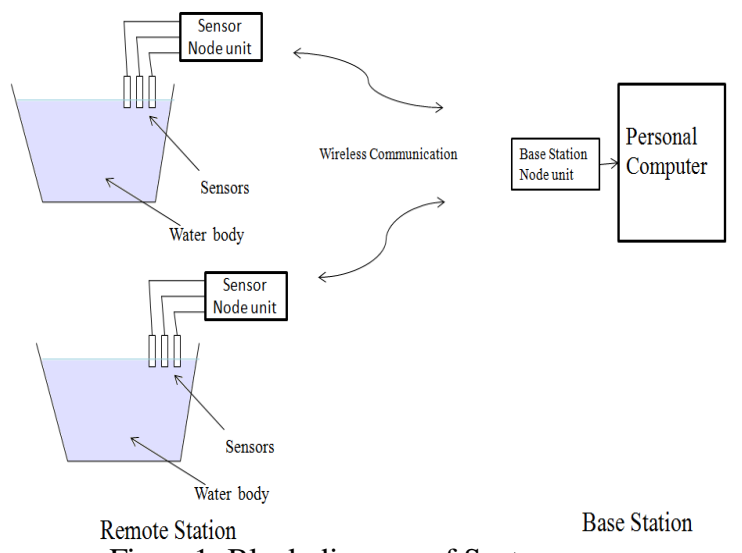

Figure1. Block diagram of System

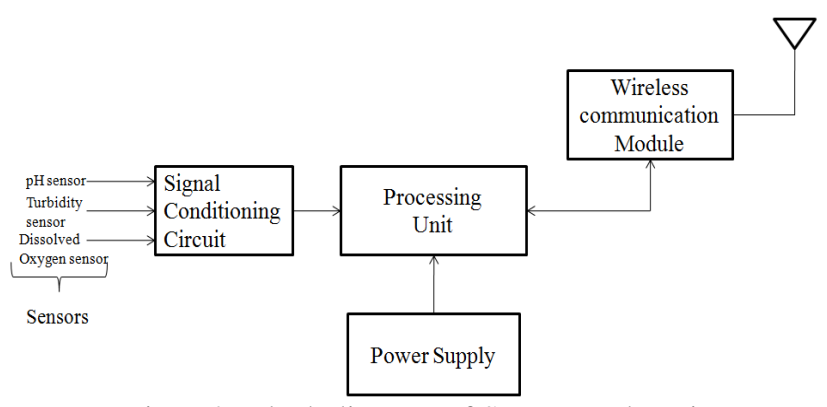

Figure2. Block diagram of Sensor Node unit 


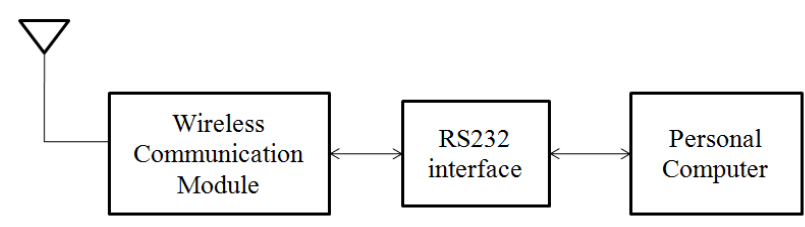

Figure3. Block diagram of base station node unit

\section{HARDWARE DESCRIPTION}

\section{A. Processor LPC 2138-}

The main function of processing module is to process or execute the instructions relating to sensing, data transmission, etc. The Processing unit converts the voltage signals from the signal conditioning circuits to numerical data, format the data into data packet, and then send the data packet to the wireless communication module for transmission.

LPC is a 32-bit microcontroller and based on ARM7TDMI-S using Von Neumann architecture. This architecture is a design for an electronic digital computer. ARM represents Advanced RISC Machine. In TDMIT stands for thumb instruction set, D is debug interface, $\mathrm{M}$ is multiplier, I for interrupt and $\mathrm{S}$ is synthesizable. It has $32 \mathrm{kB}$ of RAM and $512 \mathrm{kB}$ FLASH. There are dual 10-bit ADC and DAC. Also has two UART and I2C bus. LPC 2138 has two modes of operation; Idle and Power down. In idle mode CPU is disabled but system clock continues to operate the sources i.e. peripherals continue to operate. In power down mode CPU and system clock is disabled due to which peripherals do not operate. UART is a Universal Asynchronous Rx/Tx. UART translates the data between serial and parallel ports. I2C stands for inter integrated circuit that are used for attaching low speed peripheral devices to motherboard. ARM also supports interface to the USB. LCD display is connected to microcontroller for continuously displaying values of Temperature, humidity and soil moisture. The ARM architecture is based on Reduced Instruction Set Computer (RISC) principles. ARM used ISP i.e. in system programming. Such type of programming has the ability to program the device while installing in the system rather than programmed prior to installing. ARM has three stage pipelining; fetch, decode and execute. It uses seven modes of operation. Pipeline techniques are employed so that all parts of the processing and memory systems can operate continuously. Typically, while one instruction is being executed, its successor is being decoded, and a third instruction is being fetched from memory.

\section{B. Signal Conditioning Circuit-}

Signal conditioning circuit is used to manipulate an analog signal which is output of sensors in such a way that it meets the requirement of next stage for further processing. The output voltage of the sensors must be amplified to the appropriate levels before they can be read by the analog-to-digital converter in the next unit. The output voltage of the sensors must be amplified to the appropriate levels before they can be read by the analog-to-digital converter in the next unit.

\section{Power Supply-}

In this system there is use of total two types of DC regulated power supply, one for $+5 \mathrm{~V}$ DC used by LCD and other for $+3.3 \mathrm{~V} \mathrm{DC}$ used by LPC 2138 , Zigbee. Thus required $+5 \mathrm{~V}$ and $+3.3 \mathrm{~V}$ regulated power supply with maximum current rating $500 \mathrm{~mA}$.

The AC mains are bolstered to the transformer, which ventures down the 230 Volts to the fancied voltage. The extension rectifier takes after the transformer consequently changing over AC voltage into a DC yield and through a sifting capacitor sustains it straightforwardly into the info of the voltage controller. The regular of the voltage controller is grounded. The yield of the voltage controller is initially separated by a capacitor, and afterward the yield is taken.

D. Sensors-

1) $\mathrm{pH}$ sensor: $\mathrm{pH}$ is a measure of the acidity or alkalinity of a water solution. $\mathrm{pH}$ sensor is a passive sensor, which means there is not a need for a voltage or current source. $\mathrm{pH}$ sensors measure potential of hydrogen ion and produce a voltage. The sensor operates based on the principle that an electric potential develops when two liquids of 
different $\mathrm{pH}$ levels come into contact on opposite sides of a thin glass membrane. The sensor produces a voltage output that is linearly dependent on the $\mathrm{pH}$ of the solution it is placed in.

Measurement of $\mathrm{pH}$ can be done using glass electrodes or non glass electrodes. Here glass electrode is used. In case of glass electrodes, the $\mathrm{pH}$ sensitive element is a glass bulb that is fused to end of the glass tube. The electrode is filled with the neutral potassium chloride solution buffered $\mathrm{pH} 7$ and contains a silver chloride wire that forms the electrical connection. The reference system is located in the outer glass tube and also consist silver chloride wire in a potassium chloride solution. The $\mathrm{pH}$ value can be calculated from potential difference between reference system and measuring system. When the $\mathrm{pH}$ sensor is merged in to the solution, the hydrogen ions present in given solution are able to penetrate the boundary area of the glass membrane which results in to the charge separation. The same process occurs on the inside of the sensor with the neutral solution buffered at $\mathrm{pH} 7$ that has constant concentration of hydrogen ions. If the hydrogen ions concentration hence the $\mathrm{pH}$ value on the inside differs from the concentration of the outside a measurable potential difference works. If the hydrogen ion concentration on the inside is lower than on the outside then the measured solution is acidic. The $\mathrm{pH}$ value lower than 7 . If the hydrogen ion concentration is identical on the both side no potential difference forms and measured solution is neutral with the $\mathrm{pH}$ value 7 . The measured solution is basic if the hydrogen ion concentration inside the glass bulb is higher than the measured solution.

2) Turbidity sensor: This sensor operates on the principle that when light is passed through a sample of water, the amount of light transmitted through the sample is dependent on the amount of soil in the water. As the suspended particles in water increases, the amount of transmitted light decreases. The turbidity sensor measures the amount of transmitted light to determine the turbidity of the water.

\section{E. RS232-}

The RS232 communications is used for communications between the master unit and the PC kept beside the users table. It is realized with the help of MAX 232. Communications mode is full duplex. Maximum distance 50 feet at $19.2 \mathrm{Kbps}$. Maximum data rate is $10 \mathrm{Mbps}$ for 50 feet. Voltage levels for data 1 are $-3 \mathrm{~V}$ to $-15 \mathrm{~V}$. Data 0 are $3 \mathrm{~V}$ to $15 \mathrm{~V}$. DB9 connectors were once very common on PCs and servers. DB9 connectors are designed to work with the RS 232 serial interface standard, which determined the function of all nine pins as a standard.

\section{ZIGBEE MODULE}

Zigbee is a Technological Standard Created for Control and Sensor Networks based on the IEEE 802.15.4 specification for wireless personal area network. It is a new wireless technology that has application in various fields. Zigbee benefits are low cost and Range and obstruction issues avoidance. The main features of this standard are network flexibility, low cost, very low power consumption, and low data rate in an adhoc self-organizing network among inexpensive fixed, portable and moving devices.

There are three types of Zigbee offer in market, such XBee Series 1 that able to support up to 100 meters, XBee Series 2 that able to support until 125 meters and XBee Pro Series 1 able to support coverage up to 1.5 kilometers. The XBee Series 1 is used because the application module set up is not large. The traditional system adopts wired way wiring, which makes the system complex and expensive. ZigBee offers flexibility and mobility to save cost and energy spent on wiring.

Its ZigBee connection to the microcontroller has been implemented using a UART in both ends. This is a bidirectional channel. The ZigBee module sends the information it receives from its radio to the microcontroller so it can be processed there. The microcontroller can send packets of the data acquired by the sensors to the ZigBee module so it can be radiated and received by other nodes. The microcontroller can also send commands to the ZigBee module to configure the network.

Zigbee Technology is superior to all technologies mentioned above. Zigbee is a low cost, low power, wireless standard. Zigbee can control 254 devices at a time and has the data transfer rate of $250 \mathrm{kbps}$. It could be considered the best option to be used in wireless control and monitoring applications. Although IEEE 802.15.4 supports star and other network topologies, it operates peer to peer. However, adding network-layer specifications can introduce other capabilities. For example, adding ZigBee a top the 802.15.4 stack lets the technology work directly with other network topologies such as mesh, explained Dutton.
A. $\quad$ Algorithm for Base station node unit-
1. Start.
2. Initialization of variables LCD, ADC and Serial Communication.
3. Display the project name as WSN in Water Status Monitoring. 
4. Display characters for potential of Hydrogen, Turbidity as $\mathrm{pH} \& \mathrm{~T}$.

5. If master transfer key $* 1$ data of $S$ node 1 is displayed and if $* 2$, data from $S$ node 2 is displayed.

6. Check the condition for @ or \#.

7. If UART RBR is @. It reads the entire values form sensor node unit 1 . Send the values to HyperTerminal that will be displayed on PC.

8. If UART RBR is \#. It reads the entire values form sensor node unit 2. Send the values to HyperTerminal that will be displayed on PC.

9. Now if $\mathrm{S}$ node 1 is 1 and $\mathrm{S}$ node 2 is 1 i.e. both are on, the $\mathrm{pH}$ and Turbidity values from both the Sensor nodes are displayed.

10. If Sensor node 1 is 1 and Sensor node 2 is 0 , the $\mathrm{pH}$ value of $\mathrm{S}$ node 1 is displayed and the value for $\mathrm{S}$ node 2 is displayed as $\mathrm{xxx}$.

11. If $S$ node1 is 0 and $S$ node 2 is 1 , the Turbidity value of $S$ node 2 is displayed and the value for $S$ node 1 is displayed as Xxx.

12. If $\mathrm{S}$ node1 is 0 and $\mathrm{S}$ node 2 is 0 , the values of $\mathrm{pH}$ and Turbidity of $\mathrm{S}$ node 1 and $\mathrm{S}$ node 2 are displayed as xxx.

Thus by using polling algorithm for $\mathrm{S}$ node1, $\mathrm{S}$ node 2 and base station node the failure of any node can be easily identified. The simplicity of algorithm reduces the programming complexity.

B. Algorithm for Sensor node unit 1-

1. Start

2. Initialization of variables, LCD, ADC and Serial Communication.

3. Display project name as WSN in Water Status Monitoring.

4. Display character for Potential of hydrogen as $\mathrm{pH}$.

5. If the UART Buffer is 1. Data is read from sensor and transfers data and @ to base station node unit. Else don't read the data from the sensor.

6. If $\mathrm{S}$ node 1 receives $* 1$, the value of $\mathrm{pH}$ sensor is sent to processor that are displayed at remote station.

C. Algorithm for Sensor node unit-

1. Start

2. Initialization of variables, LCD, ADC and Serial Communication.

3. Display project name as WSN in Water Status Monitoring.

4. Display character for Turbidity as $\mathrm{T}$.

5. If the UART Buffer is 2. Data is read from sensor and transfers data and \# to base station node unit. Else don't read the data from the sensor.

6. If $\mathrm{S}$ node 2 receives $* 2$, the value of Turbidity sensor is sent to processor that is displayed at remote station.

\section{PRACTICAL IMPLIMENTATION OF THE MODEL}

The aim is to monitor the selected water parameters continuously. The modules used to implement the system are discussed earlier. Figure 4 shows base station node unit set up. On the LCD screen it shows values received from sensor node unit. Sensor node unit take the reading and send it to the base station unit via Zigbee. Figure 5 and 6 shows sensor node unit measuring $\mathrm{pH}$ and Turbidity. Sensor node unit also contains LCD to display the value of particular water parameter. GUI at base station helps to observe and save the data collected from sensor node units which are placed at remote location. When we switched on the system sensor senses the value at that time and send it to next module. So that we can get real time data at the base station and can save that data for the future reference. 


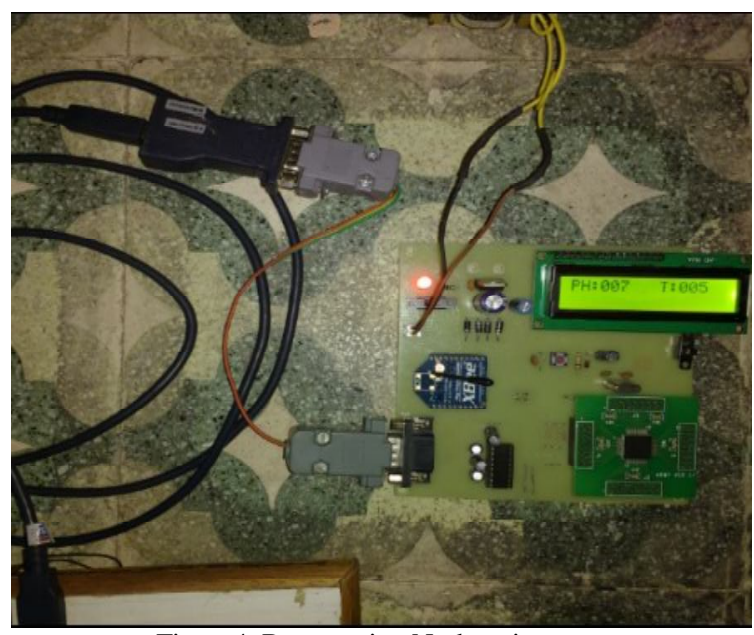

Figure4. Base station Node unit set up

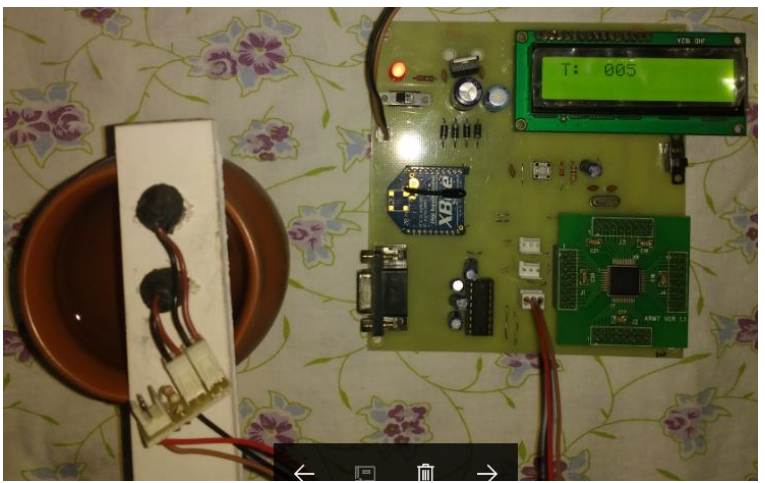

Figure5. Sensor node unit measuring turbidity of water

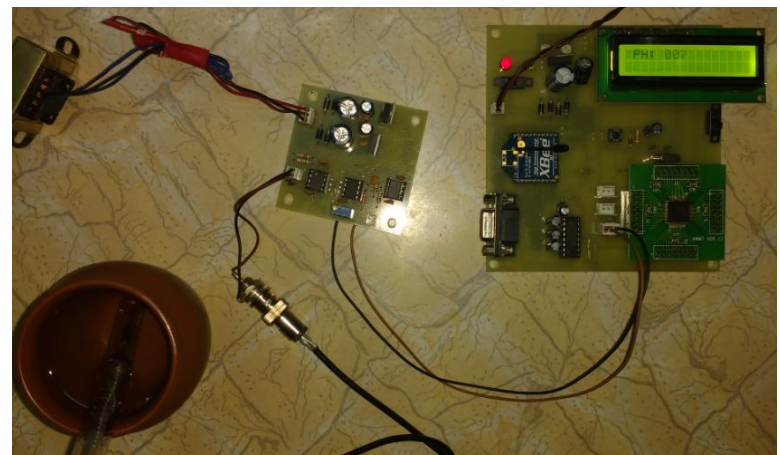

Figure6. Sensor Node unit measuring $\mathrm{pH}$ of water

\section{CONCLUSION}

The prototype hardware including the sensors for monitoring $\mathrm{pH}$ and turbidity along with circuitry is successfully implemented. The communication between the master node and computer is also implemented. The sensor data can be stored in the format of excel sheet. Compared to the traditional manual water testing, this system gives automatic reading when system is in working. The solution of ZigBee based wireless sensor network reduces the energy consumption due to its self-healing nature. This system meets the real time transmission by using the low power consumption processor LPC 2138, whose basic job is to process and collect the data, which is send to the other node through RF module.

\section{REFERENCES}

[1]. Nchimunya Chaamwe, "Wireless Sensor Networks for Water Quality Monitoring: A Case Study of Zambia", IEEE 4th International Conference on Bioinformatics and Biomedical Engineering (iCBBE), pp.1-6, 2010. 
[2]. Ruan Yue and Tang Ying, "A water quality monitoring system based on wireless sensor network \& solar power supply," IEEE International Conference on Cyber Technology in Automation, Control, and Intelligent Systems, pp.126-129, 2011.

[3]. Muhammad Azwan Nasirudin, Ummi Nurulhaiza Za'bah and O.Sidek, "Fresh Water Real-Time Monitoring System Based on Wireless Sensor Network and GSM," IEEE Conference on Open Systems, pp.354-357, 2011.

[4]. Wen-Yaw Chung IEEE senior member, Chien-Lin Chen and Jyen-bin Chen, "Design and Implementation of Low Power Wireless Sensor System for Water Quality Monitoring”, International Conference on Bioinformatics and Biomedical Engineering, (iCBBE), pp.1-4, 2011.

[5]. Theofanis P. Lambrou and Christos G. Panayiotou and Christos C. Anastasiou, "A Low-Cost System for Real Time Monitoring and Assessment of Potable Water Quality at Consumer Sites", IEEE Conference on Sensors, pp.1-4, 2012.

[6]. Patil. P.N, Sawant. D.V, Deshmukh. R.N , "Physico-chemical parameters for testing of water - A review," International Journal of Environmental Sciences, vol.3, no.3, pp.1194-1207, 2012.

[7]. Aravinda S. Rao, Stephen Marshall, Jayavardhana Gubbi, Marimuthu Palaniswami, Rechard Sinnott, and Vincent Pettigrove, "Design of Low-cost Autonomous Water Quality Monitoring System," International Conference on Advances in Computing, Communications and Informatics, pp.16-19, 2013.

[8]. Kulkarni Amruta M. and Turkane Satish M., "Solar Powered Water Quality Monitoring system using wireless Sensor Network," International Multi-Conference on Automation, Computing, Communication, Control and Compressed Sensing (iMac4s), pp.281-285, 2013.

[9]. Theofanis P. Lambrou, Christos C. Anastasiou, Christos G. Panayiotou, and Marios M. Polycarpou, "A Low-Cost Sensor Network for Real-Time Monitoring and Contamination Detection in Drinking Water Distribution Systems," IEEE Sensors Journal, vol.14, no. 8, pp.2765-2772, August 2014. 